Convergences francophones 3.1 (2016) : 22-42

http://mrujs.mtroyal.ca/index.php/cf/index

\title{
Représentations fin-de-siècle de l'homme du Nord dans La Terre de glace (1883) de Jules Leclercq
}

\author{
Marie Mossé \\ Université de Lorraine/Université du Québec à Montréal
}

Lorsque Jules-Joseph Leclercq foule le territoire islandais à l'été 1882, il réalise son "rêve le plus cher » (Leclercq 21). Honnête homme s'inscrivant dans une longue tradition de voyageurs, il vient contempler la nature islandaise et découvrir ceux qui la peuplent. ${ }^{1}$ Son itinéraire le conduit de Reykjavík à Akureyri et à la région du Myvatn en passant par les Fjords de l'Ouest, puis dans le sud de l'île (Thingvellir, site des geysers, Hékla, Oddi), et enfin à Reykir, après la traversée de la solfatare de Laugarvatn. Dans l' « Avant-propos » du récit de voyage qui en découle, La Terre de glace, Féroë, Islande, les geysers, le mont Hékla (1883), Leclercq ne prétend pas faire œuvre de naturaliste, pas plus qu'il n'affirme vouloir faire un pèlerinage sur les lieux des sagas islandaises ou écrire l'histoire du pays même si son récit de voyage fait état de ces différentes perspectives sur l'Islande. Avant toute chose, Leclercq annonce sa volonté d'objectivité, liée pour lui à un impératif de vérité :

Je raconterai l'Islande telle que je l'ai vue, et tâcherai de me garder de l'enthousiasme qui nuit à la vérité. Ce ne sera peut-être pas toujours facile. "Quand on parle de l'Islande, dit le capitaine Burton, ce vétéran des voyageurs, on est toujours en péril d'induire en erreur le lecteur, à moins qu'une convenable réflexion ne corrige une œuvre hâtive. Le sujet est, à certains égards, du type sensationnel, comme la Grèce et la Palestine; nous avons tous entendu parler dans notre enfance de ces merveilles du monde, l'Hékla et le Geyser, et comme il arrive toujours en pareil cas, nous nous sommes tous fait une Islande à nous. (Leclercq 6)

Cette volonté d'objectivité est pourtant d'emblée menacée par l'échec : en effet, l'Islande est terre de merveilles, au sens étymologique de mirabilia, chose suscitant étonnement et admiration, comme au sens de réalité surpassant les forces de la nature (Trésor de la Langue Française Informatisée). Cette terre de prodiges - le terme sera abondamment employé dans les pages qui suivent - lui fait par là

\footnotetext{
${ }^{1}$ Pour un panorama aussi exhaustif que possible des récits de voyage consacrés à l'Islande tout au long du XIX $\mathrm{X}^{\mathrm{e}}$ siècle, l'on se référera à l'importante bibliographie quadrilingue (anglais, français, suédois, islandais) établie par Daniel Chartier dans le cadre des travaux du Laboratoire international d'étude multidisciplinaire comparée des représentations du Nord, à l'Université du Québec à Montréal : A Bibliography on the Imagined North (2007); pour approfondir la question, l'on se reportera aux publications conjointes de Daniel Chartier et de Sumarliði R. Ísleifsson (Iceland and Images of the North, 2011), de François Emion et de Hanna Steinunn Thorleifsdóttir (L'Islande dans l'imaginaire, 2013), à l'ouvrage de Karen Oslund (Iceland imagined, 2011), ainsi qu'aux divers travaux d'Andrew Wawn et de Gary L. Aho, plus spécifiquement consacrés aux voyageurs victoriens, certes majoritaires, en Islande.
} 
entendre les sirènes de la fiction : de l'outrance à l'invention, en passant par la vérité arrangée et maquillée... Or, de la fiction au mensonge, écueil terrible dont se défend traditionnellement tout auteur de récit de voyage, il n'y a qu'un pas.

Pourtant, Jules-Joseph Leclercq (1848-1928) n'est pas homme à s'étonner d'un rien: ce magistrat belge, qui poursuit sa carrière jusqu'en 1921, est un voyageur au long cours, ainsi qu'un membre fondateur de la Société Royale Belge de Géographie en 1876, dont il est aussi le président et le vice-président (Biographie Royale Belge). Il écrit entre 1875 et 1928 vingt-trois relations de voyages, sans compter les nombreux articles et conférences également tirés de ses voyages. L'on citera entre autres : La Terre des merveilles : promenade au parc national de l'Amérique du Nord; Du Caucase aux monts Alaï: Transcaspie, Boukharie, Ferganah; Au pays de Paul et Virginie, Aux chutes du Zambèze : Du Cap au Katanga... Avant de découvrir l'Islande, il a notamment parcouru la Laponie, l'Amérique du Nord d'Est en Ouest, et gravi le pic de Ténériffe - il cite ce dernier souvenir dans son récit de voyage en l'Islande (Leclercq 1, 122). Ses récits de voyage - son voyage en Islande ne fait pas exception - sont remarquablement documentés : ils témoignent d'une lecture approfondie des récits des voyageurs qui l'ont précédé, et que Leclercq cite pour étayer le récit de ses expériences de voyage. Ainsi ne se rend-il pas en Islande sans avoir lu ses prédécesseurs ${ }^{2}$ - notamment les voyageurs anglais de l'ère victorienne, qui furent les découvreurs romantiques de l'Islande : entre autres, Ebenezer Henderson (1814-1815), Sabine Baring-Gould (1861), Sir Richard Burton (1872), William Lord Watts (1874), Lock, ${ }^{3}$ Arthur LeeDillon (1834), Charles William Shepherd (1862) ${ }^{4}$, John Wolley et Alfred Newton (1858), E. J. Oswald (1875), Evans, ${ }^{5}$ mais aussi le Suédois Carl Wilhelm Paijkull (1865), l'Allemand Carl Vogt (1861), cité d'après Burton sans être nommé, ou encore les travaux du philologue Richard Cleasby (Leclercq 2); il s'assure également de la connaissance des principales œuvres littéraires ainsi que des essais de géographie, de botanique, d'histoire se rapportant au pays visité, et il les cite scrupuleusement tout au long de son récit. Par cette confrontation de tous les discours, qui fait de lui un héritier de l'encyclopédisme, Leclercq entend dépasser les limites restreintes de son point de vue personnel, et appréhender au plus près la vérité d'un pays, afin de de transmettre un savoir exhaustif sur ce pays. ${ }^{6}$ Ses récits

2 J'indique entre parenthèses les dates de leurs voyages en Islande respectifs.

${ }^{3}$ Deux voyageurs nommés Lock se rendent en Islande au XIX ${ }^{\mathrm{e}}$ siècle : Charles G. Warnford Lock (1875), et William George Lock $(1878,1880)$. Or, Leclercq mentionne le premier (Leclercq 182) comme le second (240) dans son récit de voyage.

${ }^{4}$ Shepherd dont le nom est fautivement orthographié « Shepheard » par Leclercq.

${ }^{5}$ Ce voyageur n'est pas identifié : peut-être s'agit-il du compagnon de voyageur de William Morris, W. H. Evans, avec qui il se rend en Islande à l'été 1871 ?

${ }^{6}$ Le chapitre final de son récit de voyage, intitulé « La Vieille Islande », rompt avec une narration en première personne pour offrir un panorama à la fois synthétique et érudit sur l'histoire culturelle, politique, sociale...de l'Islande depuis sa colonisation jusqu'à l'âge de la domination danoise. Leclercq ne fait pas état d'un mandat de la Société Belge de Géographie, mais son récit de voyage semble s'associer étroitement à une mission savante, vraisemblablement soutenue depuis la Belgique. 
de voyage sont fidèles en cela aux objectifs de la Société Royale Belge de Géographie: ${ }^{7}$ découvrir et faire découvrir des espaces inexplorés, à des fins savantes (ce sont celles-ci qui l'emportent, dans le cas du voyage de Leclercq en Islande), mais aussi commerciales et coloniales, tout en promouvant les sciences géographiques; faire progresser la connaissance et reculer les fantasmagories. Ainsi Leclercq dénonce-t-il sans pitié les inventions dont les voyageurs du passé se sont rendus coupables dans leurs relations de l'Islande : "Vallées heureuses » (Leclercq 114), moutons à six cornes (183) ... Les chroniqueurs et critiques reconnaissent d'ailleurs la valeur de son récit de voyage en Islande (Revue de géographie commerciale 702), qui est repris par extraits en 1899 sous le titre de $L a$ Vallée des Geysers.

Ni la fascination pour les prodiges ni le goût pour les « contes merveilleux » (Leclercq 183) ne semblent donc menacer l'intégrité intellectuelle de ses récits de voyage. On ne peut cependant manquer de déceler dans la production de Leclercq, citée quelques lignes plus haut, le signe d'un rapport imaginatif au réel : tout d'abord, une inclination pour la poésie évocatrice des noms de lieux, opportunément convoqués par le souci du détail, mais aussi propres à susciter une rêverie jubilatoire par leurs sonorités dépaysantes : Boukharie, Zambèze et Katanga renvoient petits et grands enfants à l'exploration fascinée de leur globe terrestre. Ensuite, un goût certain pour les expressions stéréotypées et images d'Epinal, dont la récurrence indique une efficacité jamais démentie : la «Terre de Glace », expression certes galvaudée, réactivant littéralement l'étymologie du nom « Islande » (2), fait son petit effet ! Enfin, une constante référence à la littérature, attendue de la part d'un membre correspondant (1897) puis titulaire (1902) de l'Académie Royale des Arts, des Sciences et des Lettres : ainsi l'Isle de France (aujourd'hui île Maurice), est-elle perçue à travers l'idylle bernardinienne; de même, ce sont le Voyage en Amérique et Atala de Chateaubriand qui guident Leclercq à travers le pays des Iroquois (Leclercq, Un été en Amérique 165). Pour Leclercq, il est évident que le voyage ne se conçoit pas sans la médiation de représentations imaginaires, elles-mêmes nourries et véhiculées par la somme des lectures, savantes et/ou littéraires, qu'il a accomplies sur le sujet.

Son voyage en Islande ne fait pas exception. Leclercq est un enfant du XIX siècle, moment de redécouverte et de promotion des antiquités nordiques. De manière significative, c'est en lisant les travaux de Rasmus Bjørn Anderson ${ }^{8}$ que Leclercq rédige son récit de voyage en Islande : il cite régulièrement son essai

\footnotetext{
${ }^{7}$ Cette société savante, réunissant savants, militaires, industriels, fonctionnaires, a soutenu de nombreuses expéditions de découverte dans les années 1890, dans l'Antarctique, au Turkestan chinois, mais aussi et surtout en Afrique, de Zanzibar au Congo, dont les pouvoirs occidentaux se disputent alors la domination : cette société sera donc au cœur des expéditions coloniales et commerciales belges.

${ }^{8}$ Américain d'origine norvégienne, qui fut le tenant de la découverte de l'Amérique par les Vikings et l'instigateur du Leif Erikson Day.
} 
America not Discovered by Columbus (1874) (Leclercq, 274, 290-292), et, au moment où il découvre l'Islande, il connaît vraisemblablement déjà sa Norse Mythology (1875), qu'il traduira lui-même dès 1886 en Mythologie scandinave. Légendes des Eddas (1886). ${ }^{9}$ Il fréquente régulièrement les sagas islandaises traduites par George Webbe Dasent, et c'est en adaptant en français les traductions de sagas de Dasent, Anderson et John M. Coles qu'il introduira en France des monuments de la littérature islandaise médiévale jusqu'alors inconnus (Polet 452), rejoignant la lignée des passeurs francophones initiée par Xavier Marmier, Frédéric-Guillaume Bergmann, Edelstand du Méril, Rosalie du Puget...et perpétuée au tournant du siècle par Rodolphe Dareste et Félix Wagner... . ${ }^{10}$ Quoi d'étonnant, donc, à ce qu'il tire des sagas islandaises sa représentation de l'homme du Nord? Une représentation parfaitement conceptualisée par les personnages antinomiques de la Saga de Njál, ${ }^{11}$ Gunnar et Njál :

Les deux héros de la saga dont l'histoire se passe vers le milieu du dixième siècle sont Njál et Gunnar, deux caractères absolument différents. Njál était un homme sage et prudent le plus grand législateur de son temps : dans un siècle où la violence faisait loi, il ne portait jamais d'armes, et était respecté de tous à cause de sa science et de ses vertus. Gunnar, au contraire, brave et fougueux guerrier, se faisait craindre et admirer par sa force et son courage : c'est le Bayard de l'Islande. (Leclercq, 196)

Gunnar et Njál s'opposent terme à terme dans ce portrait en diptyque. Du côté de Gunnar, place à la force physique et morale brute : si Leclercq évacue la description physique de Gunnar, l'on ne peut ignorer la traduction de Dasent, sous-jacente, où sont mentionnées l'honnêteté de Gunnar, symbolisée par un nez droit, sa virilité -

${ }^{9}$ C'est le prélude à son ouvrage L'Islande et sa littérature (1923), qui l'inscrit dans la lignée de Xavier Marmier, le grand initiateur des études nordiques en France au XIX ${ }^{\mathrm{e}}$ siècle.

${ }^{10}$ Leclercq fait paraître cinq adaptations françaises de sagas islandaises entre 1888 et 1893 dans la Revue britannique : la Saga de Hrafnkell, la Saga des Alliés, l'Histoire de Thord le Terrible, la Saga de Thorstein, fils de Viking, la Saga de Frithiof le Hardi - ainsi que Gisli le Proscrit dans son essai L'Islande et sa littérature (1923).

${ }^{11}$ Il s'agit d'une saga qui marque Leclercq, puisqu'il intitule le chapitre $\mathrm{X}$ de son récit de voyage « Le pays de Njal ». Il ouvre ce chapitre sur le portrait comparé de Njál et Gunnar, inspiré du texte de Dasent qu'il affirme avoir lu (Leclercq, 197-198) et dont il offre une version condensée, corrigeant le romanesque échevelé de ce double portrait antithétique que Marmier avait introduit dans l'imaginaire littéraire français dès 1837 (Marmier, 274-275). Dasent, traduisant fidèlement la saga, délivre le portrait de Gunnar : "He was a tall man in growth, and a strong man - best skilled in arms of all men. ... He could swim like a seal. ... He was handsome of feature, and fair skinned. His nose was straight, and a little turned up at the end. He was blue-eyed and bright-eyed, and ruddycheeked. His hair thick, and of good hue, and hanging down in comely curls. The most courteous of men was he, of sturdy frame and strong will, bountiful and gentle, a fast friend, but hard to please when making them." (Dasent, ch. 19) ; puis celui de Njál : "Njal was wealthy in goods, and handsome of face; no beard grew on his chin. He was so great a lawyer, that his match was not to be found. Wise too he was, and foreknowing and foresighted. Of good counsel, and ready to give it, and all that he advised men was sure to be the best for them to do. Gentle and generous, he unravelled every man's knotty points who came to see him about them." (ch. 20). 
symbolisée par sa longue chevelure, attribut du guerrier - et sa force redoutable : son habileté aux armes est exprimée par une énumération d'actes extraordinaires. Ce portrait évoque le stéréotype de l'homme du Nord qui se rit de la mort et boit le sang de ses victimes dans un crâne, le pillard qui fit trembler les monastères d'Europe occidentale : « A furore Normannorum libera nos Domine ! (Boyer 1940). Du côté de Njál, le primat est donné à la sagesse et à la vie de l'esprit - son corps n'est presque pas décrit. Prudence, réflexion et lenteur évoquent en lui l'homme du Nord tel que le conçoit l'antique théorie du climat. On ne présente plus les développements de Montesquieu sur l'influence entre régime politique et raidissement du corps sous l'action du froid (Montesquieu 373-376); ni le lien établi par Mme de Staël entre climat sombre et nébuleux et littérature volontiers introspective et mélancolique (Staël, 203-212) - le tout corroboré par la théorie de Bonstetten selon laquelle le climat nocturne et hivernal oblige l'homme du Nord à se soucier de l'avenir et de sa subsistance, à privilégier la prudence au courage, et à vivre retiré en son for intérieur (Bonstetten 186-203).

Là est l'horizon d'attente du voyageur en Islande, et de son lecteur. Cet homme du Nord, bifide, évoquant un Ailleurs spatial et temporel, est autant un outil cognitif construit par la littérature et l'histoire des représentations qu'un écran susceptible d'occulter la singularité des Islandais. Quel est le destin de cette figure de l'homme du Nord? Quels en sont les autres avatars, et que cela nous enseignet-il sur l'évolution des représentations de l'Ailleurs au tournant du siècle?

\section{viking " $^{12}$ \\ L'horizon d'attente à l'épreuve du réel : le crépuscule du «mythe}

Le récit de voyage de Leclercq, avant d'en venir aux Islandais, se laisse aller à quelques pages poétiques et évocatrices sur la nature islandaise - sans doute estce la première instance à laquelle est confronté tout voyageur. Leclercq en fait un tableau vivant, au présent d'évocation.

Tout y est extraordinaire : c'est une terre de prodiges, où les feux souterrains font explosion à travers un sol glacé, où les trombes d'eau bouillante jaillissent du sein des neiges perpétuelles. Aucune autre contrée au monde n'a ce double aspect polaire et volcanique. La nature s'y montre si différente d'elle-même, qu'elle ne semble plus faite pour les êtres terrestres. Ces vallées dévastées par les volcans, ces montagnes aux cimes cratériformes, ces froides plaines ensevelies sous des nappes de lave et éclairées par la lumière étrange des silencieuses nuits crépusculaires, tout donne l'illusion de ces paysages lunaires dont le télescope nous a révélé l'aspect. L'homme ne s'y sent pas à sa place, et

${ }^{12}$ L'on emprunte à Régis Boyer cette notion de "mythe viking », par laquelle il définit la constellation des représentations de l'homme du Nord aux XVIII - XIX ${ }^{\mathrm{e}}$ siècles, laquelle repose sur de nombreux raccourcis et approximations (entre autre, la confusion entre "Viking» et «pirate $» \ldots)$. 
l'impression qu'il en rapporte est si profonde, que plus jamais il ne l'oublie (3)

Cette terre de prodiges qu'est l'Islande repose sur une nature surnaturelle - ou dénaturée ; tel est l'horizon d'attente du voyageur - en l'occurrence Jules Leclercq - et l'on peut aisément imaginer les habitants étranges qui peuplent cette contrée. Des hommes pouvant vivre là où nul autre ne le peut sont soit des surhommes, soit des hommes dénaturés : dans un cas, ils bravent la nature, dans l'autre, ils la subissent et en tirent les pires perversions. Les Islandais tels que les campe Leclercq vont de toute façon à l'encontre de la nature, et leur existence est d'emblée posée comme un mystère. Ils échappent à la compréhension du voyageur occidental, d'autant que, grimés - ou auréolés ? - par la somme des discours émerveillés ou horrifiés des voyageurs précédents, ils sont partiellement inconnus. Jules Leclercq a alors fort à faire pour cerner leur identité : « Dans un voyage en Islande, il faut, dès le premier jour, se défaire de l'habitude de faire des comparaisons avec d'autres pays ; il faut laisser de côté toute idée préconçue et s'abandonner entièrement aux impressions nouvelles » (44).

Leclercq affirme une intention tout à fait louable : celle de faire pièce aux préjugés, aux horizons d'attente qui guident le voyageur en Islande à l'âge romantique. Nul risque, donc, de voir Leclercq chercher en Reykjavík l'Athènes du Nord, ou plaquer en vain sur les gravas de Skálholt, antique évêché d'Islande, une méditation sur le passage du temps tout droit exportée des ruines hiératiques d'un forum romain ${ }^{13}$ ! Il exhorte tout voyageur, dans cette sentence injonctive, à s'ouvrir à l'altérité la plus radicale, à se laisser désorienter par une altérité spatiale, temporelle, sociale. Notons que, ce faisant, il entretient paradoxalement un effet de suspens croissant à l'égard de l'Islande, pour lui et pour son lecteur! Mais que reste-t-il au voyageur, quand les "impressions nouvelles" proposées par le voyage sont décevantes, au point, non seulement, d'apporter un cruel démenti au désir de pittoresque, mais aussi, d'escamoter l'altérité brute à laquelle se préparait le voyageur ? Ainsi Reykjavík :

C'était là Reykjavík, la capitale de l'île mystérieuse que j'avais si souvent vue en rêve! Quelle différence entre le rêve et la prosaïque réalité ! J'avais rêvé un large port d'où les hardis Vikings s'élancèrent autrefois à la découverte du Nouveau Monde ; j'avais rêvé une vieille et respectable cité tenant dignement son rang de capitale d'un pays aussi grand que l'ancien royaume de Prusse, et je ne trouvais qu'un amas de baraques en bois, éparpillées au fond d'une baie exposée à tous les vents (44)

\footnotetext{
${ }^{13}$ Penchant auquel se laissera, en revanche, volontiers aller le voyageur français Henry Labonne, épigone de Xavier Marmier, qui se rendra en Islande en 1886 et 1887, non sans citer abondamment le récit de voyage de Leclercq. Ainsi Labonne cherchant le "Capitole islandais," ou Lögberg (Labonne, 167), ou comparant la plaine d'Oddi, foyer de Snorri Sturluson, père des Eddas, aux ruines de Rome (Labonne, 76-77).
} 
Leclercq reproduit avec brio un motif devenu topique ${ }^{14}$ du récit de voyage en Islande : la déception du voyageur face à une Reykjavík misérable et dérisoire qui s'exprime ici dans une période structurée par l'anaphore de "J'avais rêvé » soulignée par l'antéposition des adjectifs, ainsi qu'une dynamique d'expansion, puis de chute brutale. Cette déception s'exprime de manière comique à travers le motif de la première rencontre du voyageur avec l'autochtone - nécessairement barbare !-, topos du récit d'exploration ici repris et retravaillé :

Cette population de Reykjavík a un aspect qui charme au premier abord: on ne voit ici que d'honnêtes physionomies ; ... . Tous les passants que je rencontrais portaient la main à leur chapeau de feutre et m'honoraient d'un salut respectueux; ceux que je croisais pour la dixième fois me saluaient une dixième fois, et $\mathrm{j}$ 'avais fort à faire pour tenir tête à un pareil assaut de politesses (49)

On constate un renversement comique : point d'incompréhension entre le voyageur et les autochtones, mais un rapport social renvoyant à un code banal, la " politesse " qui vient contrer le frisson délicieux de la confrontation avec l'Autre - une confrontation qui, pour être romanesque, doit être conflictuelle. Est-ce pour sauver in extremis le romanesque que Leclercq traduit ironiquement en termes de conflit cette politesse excessive de l'Islandais : " assaut de politesse »? Leclercq ne semble en quête que d' »impressions nouvelles » :

Sans aller en Chine ou au Japon, on a souvent vu des Chinois ou des Japonais ; mais qui donc peut se flatter d'avoir jamais vu un spécimen d'Islandais? Je me trouvais enfin au milieu des Islandais les plus authentiques, et je m'étais tant habitué à les considérer comme des gens à part, que je ne leur pardonnais pas de ressembler si fort à tout le monde. Ce qui me causait la plus cruelle déception, c'était de voir les gens se promener en pantalon et en blouse de vadmal, ${ }^{15}$ moi qui m'étais toujours représenté les Islandais dans le noble costume décrit dans les Sagas. Au lieu de chefs à chevelure et barbe incultes, vêtus de l'armure des anciens Vikings, je rencontrais de bons bourgeois exactement habillés comme tous les bourgeois ... (50)

L'on pourrait lire dans cette réaction de Leclercq un rejet du même, du proche, et une volonté louable de se confronter radicalement à l'altérité, sans médiation. L'on peut aussi y lire en filigrane une critique acerbe de la domination coloniale danoise exercée sur l'Islande, ${ }^{16}$ qui la prive de son identité propre, en en nivelant la diversité : une interprétation tout à fait recevable, quand on sait que Leclercq est

\footnotetext{
${ }^{14}$ Xavier Marmier exprime notamment sa déception face à Reykjavík (2) et Skálholt, 61-64).

${ }^{15}$ Le vadmal, remontant à l'époque des Vikings, est une étoffe filée à partir de la laine de mouton.

${ }^{16}$ L'Islande est sous domination norvégienne de 1264 à 1380, date à laquelle elle devient une province danoise, avant de regagner son indépendance en 1944, après un siècle et demi de luttes et de libéralisation progressive.
} 
amicalement reçu à Oddi par le pasteur Matthías Jochumsson (249-252), homme de lettres, mais surtout poète national islandais qui dote le pays de son hymne en 1874 - une voix patriotique à laquelle Leclercq semble se joindre dans le paragraphe final de son récit de voyage, appelant l'Islande à sortir de son long hiver pour rejoindre le concert des nations indépendantes (314). ${ }^{17}$ Néanmoins, derrière l'amoureux de l'Islande, qui ne supporterait pas de la voir amoindrie, surgit le voyageur rêveur, qu'a bercé la lecture des sagas islandaises et des Martyrs de Chateaubriand, ${ }^{18}$ et qui vient en Islande en quête du barbare Viking. Il a lui-même établi la norme de "l'authentique" : un "authentique" qui doit exhiber sa différence fondamentale, voire la mettre en scène, pour se confondre avec le pittoresque exotique. Comme Théophile Gautier, qui espère trouver dans son Tour en Belgique et en Hollande l'incarnation des femmes blondes de Rubens (Gautier 39), Leclercq se met en quête de sa propre toison d'or, la chevelure viking. Il ne résiste d'ailleurs pas à la tentation de l'avouer, avec une autodérision désarmante : « je ne leur pardonnais pas de ressembler si fort à tout le monde ». Heureusement, tous les Islandais de Reykjavík ne sont pas cruels au point de le décevoir, et les pêcheurs islandais lui offrent un curieux et dépaysant mélange de présent et de passé :

Quant aux hommes du peuple, ils rappellent bien leurs ancêtres par leur luxuriante crinière blonde et non peignée ; mais leur costume ne diffère guère de celui de nos pêcheurs; toutefois, comme il n'y a pas de cordonniers en Islande, ils ont conservé l'usage des mocassins, qu'ils taillent eux-mêmes dans des peaux de phoque ou de mouton (50)

La chevelure blonde des Vikings et les mocassins curieusement empruntés aux Inuits ou Indiens d'Amérique - Leclercq dépeignait ces derniers lors de son voyage au Québec en 1876 (Leclercq, Un été en Amérique 369, 392-393) - préservent la sensation d'altérité. La terre de prodiges qu'était l'Islande, et les êtres prodigieux qu'étaient les Islandais étaient l'objet d'une incompréhension mêlée de fascination de la part de Jules Leclercq; la terre et les êtres réels qui la peuplent sont tout aussi incompris, mais qui plus est, ils sont aussi rejetés. De manière tout à fait ironique, ce n'est pas l'Autre que rejette Jules Leclercq en la personne de ses contemporains islandais, c'est le Même - une parenté insupportable, qui abolit la distance spatiale et temporelle nécessaire au fantasme, à la projection. Il est alors possible de voir en Leclercq un "proxénète de la Sensation du Divers » (Segalen 34), selon le mot terrible que Victor Segalen adresse à Pierre Loti dans les notes de son Essai sur l'exotisme. On pense au désespoir d'un Loti bercé par la lecture des Mille et Une Nuits, dans ses Suprêmes Visions d'Orient, face au sultan Mahomet V, vêtu « d'un complet gris » comme « un quelconque bourgeois de France» (Loti 1399). Mais, là où Loti se complaisait dans l'évocation, voire dans la mise en scène d'un Orient

${ }^{17}$ Cet appel est d'ailleurs relayé par le préfacier de La Vallée des Geysers, Charles Simond (Leclercq Vallée des Geysers, 6).

${ }^{18}$ Songeons notamment à la description grandiose et d'inspiration ossianique des cavaliers francs de Pharamond, tout en armures (Chateaubriand 200). 
rêvé et inexistant, Leclercq, quant à lui, est conscient de ses propres illusions, et n'hésite pas à user de l'autodérision pour évoquer ses espoirs déçus de voyageur rêveur - et l'on doit alors relire d'un autre œil l'emphase désormais suspecte de sa déception face aux habitants de Reykjavík. Il ne cache pas le caractère égoïste et potentiellement risible de son obsession pour l'Islandais authentique - un Islandais vêtu à la mode danoise ne peut en être un, il se doit d'arborer armure et casque à cornes - et exerce sur cette obsession un regard critique, mais y renonce-t-il pour autant ? Le Viking, fût-il repoussé dans l'orbe du rêve, réapparaît de manière fugace dans les paysages islandais, par hypotypose, en guise de consolation :

Quelle étrange impression j'éprouvai lorsque je sentis sous mes pieds le sol du Lögberg, ce forum des anciens Islandais! Le glorieux passé de l'Islande revivait tout entier dans mon imagination, et il me semblait avoir reculé de plusieurs siècles. Je voyais affluer à Thingvalla le peuple venu de tous les points du pays pour assister à la foire annuelle qui se tenait pendant la réunion de l'Althing ${ }^{19}$. Cette plaine, qui n'est plus aujourd'hui qu'un désert morne et silencieux, se couvrait alors de milliers de tentes et retentissait d'un bout à l'autre du hennissement des chevaux. Les puissants chefs étaient revêtus de leur armure et escortés d'une suite nombreuse. (Leclercq 133)

Mais le rêve est bientôt dissipé par la découverte du parlement islandais moderne, décrit comme "mesquin" (272) et si peu digne des anciennes assemblées du Lögberg. La plus grande épreuve du voyageur est alors non plus de surmonter la différence radicale, mais de faire le deuil d'une altérité rêvée, pittoresque, conçue par et pour lui, afin de pouvoir admettre celle, véritable, de l'Islandais réel. Un voyageur peut-il cependant renoncer au pittoresque ?

\section{Une altérité choisie : Leclercq, ethnographe ou rêveur?}

C'est à une fracture entre le réel et l'imaginaire que Jules Leclercq se confronte. L'altérité de l'homme du Nord s'exprimait en termes spatiaux et temporels: un homme façonné par son climat extrême, fort et vertueux, ou mélancolique, un homme surgi du passé légendaire des sagas islandaises, et aurait « dans les veines du sang de Viking » (305). Face à un Islandais proche et banal, deux options s'offrent à Leclercq : chercher la véritable essence de l'Islandais (puisqu'elle n'est plus définie par le Viking), en observant les êtres qui l'entourent et en en saisissant l'irréductible singularité ; ou faire appel à de nouvelles stratégies discursives, propres à réinstaurer une "impression" d'exotisme dans la perception de l'Islandais.

\footnotetext{
${ }^{19}$ Le Lögberg, littéralement "Montagne de la Loi," est le promontoire surplombant Thingvellir, lieu historique du sud-ouest de l'île, qui accueillit chaque été les séances du Parlement islandais, l'Althing, entre le $\mathrm{X}^{\mathrm{e}}$ et le $\mathrm{XIII}{ }^{\mathrm{e}}$ siècles.
} 
La première possibilité semble confirmée par le mouvement général de l'itinéraire et du récit de voyage de Leclercq : Reykjavík est certes le lieu qui cristallise toutes les projections du voyageur, mais c'est aussi la porte de l'Islande, lieu intermédiaire, ouvert sur l'extérieur de l'Islande. Par conséquent, la progression de Leclercq à travers l'Islande d'une part, et d'autre part, le degré d'étrangeté de ses habitants, le sentiment de décalage du voyageur et l'authenticité de la confrontation avec l'Autre doivent être proportionnels. C'est le cas, comme nous allons le voir. Cependant, il faut nuancer cette proposition: de fait, plus Leclercq s'aventure dans l'Islande, plus ses observations anthropologiques sont guidées par des choix, d'ordre personnel et idéologique. Il sélectionne ses "spécimens d'Islandais" comme le ferait le naturaliste, mais aussi le collectionneur d'un cabinet de curiosités. Ainsi le guide Zoega, personnage haut en couleur voué à l'immortalité littéraire par tous les récits de voyage de la seconde moitié du XIX ${ }^{\mathrm{e}}$ siècle, et guide officiel du roi du Danemark lors de sa visite en Islande en 1874, estil rapidement passé sous silence : outre le fait qu'il n'organise désormais plus que de loin les expéditions touristiques, il «parle l'anglais comme un gentleman du West-End » (16)! Jon Jonssen, ${ }^{20}$ guide dont Leclercq s'offre les services, dans la région de Thorsmörk, semble cadrer davantage avec le stéréotype du guerrier islandais, dont le voyageur ne parvient jamais tout à fait à se défaire : « Jon Jonssen me fait songer aux anciens héros scandinaves : sa carrure colossale, sa large figure, sa crinière de lion, sa barbe rousse, ses yeux bleus et jusqu'au timbre de sa voix, tout rappelle en lui le type de Gunnar, tel qu'il est dépeint dans la saga ; je ne serais nullement surpris qu'il descendit de lui en droite ligne » (204).

Il annonce également la méthode cognitive qui sera celle de Leclercq pour appréhender les Islandais : le recours à la littérature, et aux stéréotypes efficaces qu'elle forge - stéréotypes garants d'exotisme qui doublent la perception du réel. Quant aux pasteurs islandais qui accueillent Leclercq et son guide pour la nuit au cours de sa traversée d'Islande, ils se ressemblent tous : pauvres, honnêtes, savants, voués à l'étude, et dotés d'une fille belle et serviable qui aide le voyageur à prendre possession de ses pénates. Les entrevues avec ces Islandais pourtant non moins islandais que les autres se confondent les unes avec les autres dans la mémoire du lecteur - à l'exception de la rencontre avec Matthías Jochumsson. Seules demeurent les expériences de voyage, qui ont tiré le voyageur de sa zone de confort pour le confronter à la différence radicale - ou du moins, seules demeurent les expériences jugées dignes d'être racontées, réécrites, mises en littérature.

Ce qui intéresse véritablement Jules Leclercq, ce sont les « indigènes » (195). Ce terme, récurrent tout au long du récit de voyage, est à entendre selon ses deux principaux sens : 1/ qui est originaire du pays où il habite, 2/ qui appartient à la population implantée dans un pays avant sa colonisation, par opposition aux colons (Trésor de la Langue Française Informatisée). Le second sens contribue

${ }^{20}$ Du moins est-ce l'orthographe choisie par Leclercq : le véritable nom de cet Islandais serait Jón Jónsson. 
évidemment à instaurer une scission, une différence de statut -voire de natureentre le voyageur occidental et l'Islandais, ce qui réinstaure une relation de confrontation culturelle. Lorsqu'il s'agit de dépeindre les "indigènes," Leclercq oscille entre sa volonté d'exactitude et d'exhaustivité qui fait de lui un précurseur des ethnographes, et sa fascination pour l'exotisme. Notre hypothèse est la suivante : la rencontre avec les indigènes, qui se décline selon plusieurs modalités, de la plus euphorique à la plus dysphorique, s'exprime dans le récit de voyage de Leclercq à travers la conjonction paradoxale d'une description tendant au réalisme et à l'objectivité et du recours répété à des modèles discursifs stéréotypés, empruntés à la littérature de voyage (le bon sauvage, le barbare...).

Lorsque l'advenue de l'altérité coïncide fortuitement avec un horizon d'attente façonné par les lectures romanesques de Leclercq, la rencontre avec l'indigène est pour le voyageur source d'enchantement et d'émerveillement. Ainsi, la rencontre de cette jeune amazone, à Keldur :

Johannes lui demande si la rivière Vestri-Ranga est praticable, et aussitôt elle s'offre à nous accompagner jusqu'au gué. Elle saute avec l'adresse d'une écuyère sur un cheval qu'elle ne prend pas la peine de seller, et la voilà galopant en tête de la cavalcade, à la façon des intrépides amazones d'Havaï, astride, comme disent les Anglais, serrant les flancs de sa monture avec ses jambes fines qu'enveloppent de longs bas noirs et de jolis mocassins. Au bout d'une heure, nous arrivons au bord de la rivière, que nous trouvons considérablement gonflée par la fonte des neiges ; il me paraît difficile de la passer à gué, tant elle est tumultueuse ; mais la jeune Islandaise lance sans aucune hésitation son poney dans l'eau et nous fait signe de la suivre ... (Leclerc 193)

La jeune femme, dépeinte avec force détails, se double d'un être aussi providentiel et fugace qu'une fée médiévale, exotique par son caractère composite : elle évoque les Indiens d'Amérique par ses mocassins, ainsi que par le rapport intime et mystérieux qu'elle semble entretenir avec la nature par le biais de son cheval. Le rapport fusionnel des Islandais avec leurs chevaux est néanmoins souvent mentionné par Leclercq, notamment pour y opposer avec humour ses médiocres prestations équestres - et cette notation réaliste exprimée sur le ton de l'autodérision, invoque parfois une semi-bestialité légendaire : « les Islandais, qui passent toute leur vie à cheval, ne comprennent guère que tous les hommes ne soient pas des centaures » (146). La jeune cavalière évoque enfin les femmes intrépides et farouchement indépendantes qui s'illustrent notamment dans la Saga de Laxdaela (Boyer, Sagas islandaises 388-571) : la belle et terrible Gudrun ; Aude-la-TrèsSage, meneuse d'hommes, des Hébrides à l'Islande ; ou encore la druidesse Velléda de Chateaubriand. Autre nuance dans le kaléidoscope des représentations positives des Islandais, la rencontre avec les fidèles d'une petite église islandaise, après la messe : 
bientôt arriva l'heure touchante des adieux. Tous, hommes, femmes, donnèrent un bruyant baiser au prêtre, qui à son tour baisa tout le monde sans distinction; ce furent des baisements à n'en plus finir. Tout cela se faisait gravement, solennellement ; les Islandais s'embrassent d'un air triste, comme s'ils ne devaient jamais se revoir, comme s'ils se sentaient sans cesse en danger de périr au milieu de leurs volcans. Que j'aime ces braves gens, en dépit de leur écorce un peu rude et de leur horreur des ablutions! Leurs mœurs ont quelque chose de si patriarcal, de si biblique! (Leclercq 181)

Dans cet éloge sans réserve est réactivée l'image du bon sauvage, libre, ignorant des us et coutumes de la société que sont les ablutions, mais empreint d'une pureté édénique. Ici est bien évidemment sous-entendue la corruption de l'homme civilisé, qui n'a d'égal que son supposé raffinement. Leclercq poursuit l'analogie entre bon sauvage et Islandais, suggérant que ce dernier appartient à un passé révolu et regretté :

Ce qui d'ailleurs distingue l'Islandais de la plupart des peuples modernes, c'est le peu de cas qu'il fait de l'art de faire fructifier l'argent, comme le témoigne assez l'absence complète de banquiers sur tout le territoire de l'île. Accoutumé à la vie pastorale, et n'ayant d'autres biens que ses pâturages et son bétail, le fermier islandais se contente de posséder assez de moutons pour ne manquer ni de laine pour ses vêtements, ni de viande et de laitage pour sa subsistance. Comme la population est clair-semée, il ne connaît point les inconvénients qui résultent de la division de la propriété; il est généralement propriétaire d'une assez vaste étendue de terres qu'il exploite suivant les mêmes procédés que ses ancêtres (312)

L'évocation d'une vie pastorale et idyllique se double d'une condamnation du droit de propriété et de l'argent aux accents rousseauistes (Rousseau, 100-101). Leclercq dépeint l'état intermédiaire entre nature et société, fragile, ténu, susceptible d'être brisé par l'intrusion de la civilisation - voyageurs et marchands. ${ }^{21}$ Brisé, il l'est d'ailleurs, comme le dénonce Leclercq :

L'ivrognerie et la saleté sont les deux péchés capitaux des Islandais. J'ai souvent rencontré des paysans dans un tel état d'ébriété, que je me demandais comment ils pouvaient se tenir à cheval. Leur ivresse n'est pas méchante, mais elle les plonge dans un hébétement qui leur ôte toute conscience de leurs actes. Lorsqu'ils se rendent chaque année aux

${ }^{21}$ Ce n'est pas la première fois qu'intervient une telle condamnation de la corruption de l'homme du Nord par la civilisation : Ampère, dans ses Esquisses du Nord, vante l'honnêteté proverbiale des Norvégiens, constatant que l'unique crime survenu dans un village montagnard s'est produit par l'entremise de voyageurs, qui ont tenté les Norvégiens par l'étalage de leurs richesses (Ampère 107108). 
stations de la côte pour faire leurs échanges, les marchands, avant de conclure leurs affaires, ont bien soin de les griser, pour leur acheter ensuite leurs articles à un prix dérisoire. Et le malheureux paysan s'en retourne chez lui plus pauvre que jamais (267)

Cette évocation rappelle l'image désormais topique des sauvages tahitiens ravagés par l'abus d'alcool - la tonalité véhémente de Leclercq est aussi celle des condamnations formulées par le vieux Tahitien de Diderot (Diderot 14-17). Les modèles discursifs topiques des récits de voyage sont convoqués pour exprimer la réalité de la société islandaise que Leclercq dépeint en connaissance de cause : une société frugale, où le troc est encore fréquent, et où l'entraide, l'hospitalité et la relation à l'autre sont réglées depuis des siècles par le Hávamál, traité de savoirvivre (Boyer, Edda poétique 169-202), mais où l'alcoolisme et la misère, unanimement dénoncées par les voyageurs du $\mathrm{XIX}^{\mathrm{e}}$ siècle, font bel et bien des ravages... L'anecdote du malheureux paysan escroqué lors de son commerce annuel avec les marchands étrangers est récurrente dans les récits de voyage des prédécesseurs de Leclercq. La réalité trouve ici à s'exprimer à travers le recours à une riche mémoire intertextuelle.

A ces représentations positives de l'Islandais, où la volonté de précision et d'objectivité de la description se teinte insensiblement de rêveries romanesques et de nostalgie rousseauiste de l'âge d'or, il convient d'opposer les expériences de voyage dysphoriques, où l'Islandais est source d'effroi et de dégoût, sans qu'aucune instance étrangère ne se soit rendue responsable de sa corruption. Ainsi la description précise des mœurs des paysans islandais :

ces bancs servent de sièges pendant le jour et de lit pendant la nuit, exactement comme chez les Kabyles. Les Islandais mangent même dans leur lit, toujours comme les Kabyles, et il m'est arrivé quelquefois de trouver sous mes couvertures des têtes de morue et autres débris de repas oubliés par quelque indigène.

A l'heure du repas, la badstofa offre un curieux coup d'œil : il n'y a ni table, ni chaises, ni assiettes, ni couteaux, ni fourchettes; les Islandais ignorent ces raffinements de la civilisation. Du fond de leurs lits ils attaquent avec les doigts la morue séchée et mangent avec de grandes cuillers de bois leur $\mathrm{skyr}^{22}$, plat national qui joue chez eux le même rôle que le couscoussou chez les Arabes.

... comme les Islandais n'ont point de combustible, ils ignorent l'usage des poêles ... (99-100)

C'est un univers renversé qui est ici dépeint, tout entier sous-tendu par l'opposition traditionnelle entre nature et culture : confusion des espaces de vie, ignorance du combustible rappelant en filigrane l'ignorance du feu, dont dépend la maîtrise des

${ }^{22}$ La badstofa est la pièce à vivre d'une ferme islandaise, et le skyr est une sorte de fromage blanc. 
techniques depuis le mythe de Prométhée. Il faut également souligner le rôle de l'analogie entre Kabyles et Islandais, récurrente dans le récit de Leclercq, et même théorisée par lui, comme l'atteste cet extrait :

Tous ceux qui ont voyagé en Islande ont été frappés des nombreuses analogies que cette contrée perdue au milieu des glaces polaires offre avec l'Orient. ... Quant aux habitants, les ressemblances sont plus saisissantes encore; ils rappellent les Orientaux par leurs mœurs nomades ... . Comme les Arabes, ils regardent les rites de l'hospitalité comme sacrés, ont horreur de la marche à pied, abusent du café et du laitage, sont très-expansifs dans leurs salutations, se servent de la bouse des bestiaux en guise de combustible, mangent avec les doigts et se mouchent de même. Ce qui les rapproche le plus des peuples de l'Orient, c'est encore leur saleté, qui n'a guère changé depuis le temps de madame Ida Pfeiffer ; la célèbre voyageuse trouvait les Islandais, sous ce rapport, inférieurs même aux Bédouins et aux Arabes (254-255)

Cette analogie est certes destinée à éclairer la réalité décrite par Leclercq, en ramenant de l'inconnu à du connu: si l'Islandais est peu connu du public francophone, le Bédouin, figure récurrente des voyages d'Orient romantiques, l'est davantage. Cependant, elle permet, paradoxalement, de réinstaurer de l'exotisme là où il n'y en a pas a priori: l'Islande se superpose à d'autres contrées traditionnellement exotiques, et renvoie le lecteur à un horizon d'attente bien connu : qui dit Bédouin dit vie dans la nature, nomadisme, pauvreté... Il n'en faut guère plus pour bannir les Islandais de l'humanité, et les placer dans un rapport d'infériorité par rapport au voyageur qu'est Leclercq. En marge de la société et de la nature - ce qui les rend fascinants à observer pour le lecteur - ils sont exclus du progrès historique, confinés dans une altérité temporelle :

Si primitive que soit la selle de charge islandaise, les indigènes n'ont encore rien trouvé de mieux. C'est en vain qu'on voudrait introduire chez eux la selle de charge des Otagos, dont les Anglais se servirent dans l'expédition d'Abyssinie, et qui a été reconnue excellente par tous les explorateurs qui en ont fait usage ; les Islandais sont ennemis de la nouveauté : leurs ancêtres du moyen âge disposaient leurs bagages sur une selle de tourbe, ils procèdent comme leurs ancêtres et continueront à le faire sans que rien puisse les décider à se départir de la vieille routine (12)

Ici est discrètement réinstaurée la posture du voyageur civilisateur, qui apporte la lumière aux peuples découverts, malgré eux. La différence radicale de nature entre le voyageur et le sauvage est consommée dans ce compte-rendu dégoûté des mœurs alimentaires islandaises :

je ne saurais avoir aucune considération pour des gens qui peuvent digérer les horribles plats dont j'ai fait la connaissance chez 
mon hôte, et parmi lesquels je citerai la chair de requin séchée et la graisse de baleine. Surmontant mes répugnances, $\mathrm{j}$ 'ai voulu goûter ces mets d'une haute nouveauté : j'en ai eu l'estomac bouleversé pendant huit jours. Les Islandais ont des raffinements qui nous font dresser les cheveux sur la tête ; ils ne mangent la chair du requin qu'au bout d'un an, prétendant qu'elle gagne à vieillir, comme le wiskey. Je laissais aux indigènes ces vilains plats ... (73-74)

L'ironie de Leclercq (soulignons l'emploi antithétique de "raffinements ») ne suffit cependant pas à escamoter sa jouissance discrètement morbide du spectacle : les mets islandais, pour être répugnants, n'en sont pas moins inhabituels, et donc exotiques; et Leclercq n'hésite pas à accentuer le caractère dégoûtant de son expérience en maniant l'hyperbole et en accumulant les petits faits vrais et anecdotes qui font le bonheur des curieux. Un autre exemple, cet extrait rapprochant insensiblement l'homme et la bête :

C'est par leur contact continuel avec les chiens que les Islandais contractent le germe de ces redoutables parasites; ils laissent les chiens coucher avec eux dans leur lit pendant la nuit, et ils poussent la malpropreté et l'insouciance jusqu'à leur donner à lécher leurs ustensiles de cuisine au lieu de les laver. ... Il est même avéré que beaucoup d'Islandais contractent le ténia en s'administrant les excréments du chien en guise de remède interne contre une foule de maladies (101)

L'horreur de l'évocation, fabuleuse autant que répugnante, n'est pas sans rappeler le goût médiéval des mirabilia du Nord. ${ }^{23}$ C'est en effet ce procédé discursif qui habite le récit de voyage de Leclercq, et qui culmine paradoxalement dans des notations d'un réalisme indubitable: les voyageurs sont nombreux à évoquer l'hospitalité qu'ils reçoivent -bon gré mal gré- du misérable paysan islandais, son improbable pitance et le ténia qui le dévore ; ils sont nombreux aussi à parvenir aux mêmes conclusions : l'Islande du XIX ${ }^{\mathrm{e}}$ siècle est un pays oublié par l'Histoire, accablé par la domination politique et économique danoise comme par les éruptions et les séismes qui l'ont affligé au cours des derniers siècles. Mais l'Islande profonde est aussi, dans sa désolation hyperbolique et sa position en marge de la civilisation occidentale, le dernier refuge de l'Autre. Là gît l'ambiguïté fondamentale du texte de Leclercq, entre réalisme et rêve, entre indignation et fascination.

On le constate à travers ce kaléidoscope d'Islandais, Leclercq déplace le curseur, d'une sauvagerie infra-humaine, celle du barbare ignorant de toute technique, à une sauvagerie de bon aloi, de proximité avec la nature. Il loue cette douce sauvagerie comme il condamne la sauvagerie bestiale, mais les deux ont

${ }^{23}$ On songera aux us et coutumes supposés des Irlandais (zoophilie, notamment), retracés avec force détails par l'auteur médiéval Giraud de Barri dans sa Topographia Hibernica, et propres eux aussi à faire vaciller la frontière entre humanité et bestialité (Boivin). 
également droit de cité dans son récit : d'abord, parce que Leclercq vise à offrir des Islandais, de l'Autre, une représentation aussi exacte et exhaustive que possible, mais aussi parce que tout vaut mieux que la douce banalité des habitants bourgeois de Reykjavík - pourtant tout aussi islandais ! Les extraits cités sont remarquables par l'attention portée au réalisme de la description, autant que par la résurgence constante d'un horizon d'attente, l'Ailleurs et l'Autre, qu'il s'agit pour Leclercq de préserver à tout prix. De fait, les propos à vocation ethnographique de Leclercq se fondent sur des observations de médecins et de savants, mais n'échappent pas au goût -universellement partagé ?- de l'étonnant et du prodigieux. On le comprend bien vite, il n'y a point de description ethnographique précise sans la fascination irrationnelle du voyageur occidental pour des mœurs qui lui sont étrangères, sauvages : elle se fait malgré, ou par cette donnée de fait. Leclercq se veut un voyageur observateur, en situation d'extériorité, capable d'étonnement, de surprise... Cet étonnement, cependant, exige d'être balisé par un cadre littéraire et théorique bien connu, tant dans sa perception que dans le discours qui en est tenu. Il est décliné par degrés, se fondant sur une représentation de l'Ailleurs sous-tendue par l'opposition conceptuelle entre sauvage et civilisé, où l'Islandais peine à trouver sa part. Trop civilisé, il est banni du récit de voyage ; sauvage, le voilà prisonnier, de la vertu du bon sauvage ou de l'incorrigible perversion du barbare. Autant de masques qui lui sont apposés, disséminant son identité, dépeignant de lui une réalité fragmentée et contradictoire, une mosaïque de stéréotypes ?

\section{Conclusion : vérités de la fiction?}

Jules Leclercq est fidèle à ses prédécesseurs, dont il reproduit les stéréotypes : 1'opposition conceptuelle entre bon sauvage et barbare, récurrente dans son récit, semble notamment être un outil cognitif propre à permettre l'appréhension d'un peuple encore mal connu du public francophone - si les récits de voyage en Islande sont légion dans le domaine anglo-saxon au XIX ${ }^{\mathrm{e}}$ siècle, ils font figure d'exception dans le domaine francophone. L'on aurait tort, cependant, d'attribuer à cette opposition conceptuelle une quelconque valeur édifiante : ce n'est plus le miroir critique que les récits de voyage se plaisaient à tendre à la société occidentale pendant les Lumières; ce n'est pas non plus un éloge sans réserve de la civilisation moderne à l'âge du positivisme. Il s'agit plutôt d'une rêverie fin-desiècle sur la possibilité d'un Ailleurs géographique et historique, que peuplerait un Autre radical ; d'un adieu, lourd de regrets, aux stéréotypes, machines à rêves dont Jules Leclercq démonte plus ou moins les rouages -question de préférences personnelles ? Il épargne davantage les sauvages que les Vikings! De ce tour d'horizon, il ressort que l'Islande est la suprême terra incognita de l'âge moderne, l'ultime conservatoire d'un exotisme séculaire - mais aussi le laboratoire du discours contemporain, encore balbutiant, sur l'Ailleurs...

En effet, l'originalité de Leclercq consiste à réfléchir sur le voyage, sur la question de l'exotisme, sur l'horizon d'attente du voyageur, non sans autodérision. Il est à la fois la proie et l'observateur critique des lubies du voyageur, introduisant 
dans son récit une dimension réflexive par la distance de l'humour. Ce va-et-vient entre le laisser-aller irrésistible aux représentations imaginaires et la ressaisie intellectuelle de ces mêmes représentations dote de profondeur le récit de voyage de Jules Leclercq. Il réaffirme aussi la légitimité de la littérature en tant qu'outil cognitif permettant la saisie du réel.

Sans doute Leclercq ne touche-t-il jamais autant à la vérité des habitants de l'Islande que lorsqu'il conjoint les antiques sagas islandaises et sa propre expérience de voyageur pour évoquer leur indéfectible attachement à leur île - autre topos du récit de voyage en Islande :

Rien n'est plus touchant que l'attachement des Islandais pour leur patrie ; ceux qui émigrent dans des régions plus favorisées la regrettent toujours, et leur constant désir est de la revoir quand ils auront amassé un pécule. On m'a conté que l'un d'eux, après plusieurs années de séjour en Europe, fondit en larmes un jour qu'il entendit parler sa langue maternelle, et s'embarqua sur le premier bateau faisant voiles pour Reykjavík. L'Islandais, comme le Lapon, l'Esquimau et le Samoyède, vit dans un climat qui lui appartient en quelque sorte; il y conforme ses mœurs, et préfère à nos vertes campagnes ses déserts de lave, ses glaciers et ses volcans neigeux; il est né au milieu d'eux, et ses yeux sont habitués à des paysages qu'il ne trouve nulle part ailleurs ; aussi se plaît-il à répéter avec conviction ce vieux proverbe islandais : Island er hinn besta land sem solinn skinnar uppa : ${ }^{24}$ " L'Islande est le meilleur pays sur lequel brille le soleil, » C'est se faire une grande illusion que de faire consister le bonheur uniquement dans la possession du bien-être ! (Leclercq 26)

Le propos, fondé sur le récit d'une expérience, glisse du discours ethnographique Leclercq réfléchit en termes de nationalités (Islandais, Samoyède...) - à l'abstraction poétique : 1'Islande, de type, devient allégorie, figé par un présent de vérité générale dans l'amour de son pays. Sa parole, islandaise dans le texte, est gravée dans la perfection formelle de la sentence. Il touche ainsi à l'immortalité discrète que lui offre le récit de voyage, venant à la suite d'un autre Islandais amoureux de son île - et devenu un monument de la littérature de saga : le héros Gunnar, condamné à l'exil sur la décision de l'Althing :

La saga est ici d'une grandeur héroïque. « Empêche-moi, disait Gunnar à son frère Kolskegg, de regarder derrière moi, de peur que mon courage ne faiblisse. » Tout à coup son cheval tomba lourdement avec lui, et quand il se releva, ses yeux rencontrèrent sa maison, ses champs couverts de récoltes, et son cœur fut sur le point de se briser dans sa poitrine. « Le beau pays ! dit-il. Le blé croît sur les bords de la rivière,

\footnotetext{
${ }^{24} \mathrm{Ou}$, si l'on respecte l'orthographe islandaise en vigueur: Ísland er pað besta land sem sólin skín
} uppá. 
les prairies sont fauchées, toute la campagne est prête pour la moisson. Non! je ne puis aller plus loin. Je veux terminer ici mes jours. » Et il revint rapidement sur ses pas, malgré l'arrêt de proscription qui pesait sur sa tête, et en vertu duquel tout homme pouvait légitimement le tuer ; ses ennemis prirent soin que ses jours ne fussent plus de longue durée (197-198)

La juxtaposition entre le réel et la littérature, entre le présent et le passé, s'opère à travers l'anecdote touchante de l'Islandais en instance d'exil. Elle auréole les Islandais de Leclercq de l'héroïsme de Gunnar, tout en offrant à Leclercq la meilleure compréhension possible du pays qu'il visite, transcendant la multiplicité éparse des représentations de l'homme du Nord. Leclercq donne alors à voir l'étonnant Islandais, énigme non résolue aux yeux des voyageurs. Mais cet Islandais n'habite plus une terre de prodiges; il aime sans condition une terre hostile et rude, dont il révèle par son amour les beautés insoupçonnées.

\section{Bibliographie}

Aho, Gary L. "'Með Ísland á heilanum' : Islandsbæekur breskra ferđalanga 1772 til 1897." [version anglaise : "'Iceland on the Brain' : British Travellers to Iceland from 1772 to 1897."] Skírnir 167, 1 (1993) : 205-258. Imprimé.

Ampère, Jean-Jacques. Esquisses du Nord, in Littérature et Voyages. Paris : Paulin Libraire-Editeur, 1833 ( $1^{\mathrm{e}}$ édition). Imprimé. Consultable en ligne : https://books.google.ca/books?id=W5kTAAAAQAAJ

Anderson, Rasmus Bjørn. America not Discovered by Columbus. A Historical Sketch of the Discovery of America by the Norsemen in the Tenth Century. Chicago : S. C. Griggs and Company, 1874 ( $1^{\mathrm{e}}$ édition). Consultable en ligne : https://archive.org/details/americanotdiscov02ande.

---. Norse Mythology. The Religion of our Forefathers, Containing all the Myths of the Eddas. Chicago et Londres: S. C. Griggs and Company/Trübner and Co., $1875 \quad\left(1^{\mathrm{e}}\right.$ édition). Imprimé. Consultable en ligne: https://archive.org/details/norsemythologyo00ande.

Anonyme. «Dr Henry LABONNE. L'Islande et l'archipel des Farcer. Paris, Hachette, 1888, in-16, xx-399 pages ; 57 gravures et une carte ; 4 francs ». Bulletin de la Société de géographie commerciale de Bordeaux, 1888, 702703. Imprimé. Consultable en ligne : https://books.google.ca/books?id=s93NAAAAMAAJ.

Boivin, Jeanne-Marie. L'Irlande au moyen âge, Giraud de Barri et la " Topographia Hibernica » (1188). Paris : Champion, 1993. Imprimé.

Bonstetten, Charles-Victor de. L'Homme du Midi et l'Homme du Nord, ou l'Influence du Climat. Genève et Paris : J. J. Paschoud, 1824 ( $1^{\mathrm{e}}$ édition). 
Imprimé. Consultable en ligne : http://gallica.bnf.fr/ark:/12148/bpt6k107872r.

Boyer, Régis (trad. et éd.). L’Edda poétique. Paris : Fayard, 2014 (1édition : 1992). Imprimé.

---. Le Mythe Viking. Paris : Editions du Porte-Glaive, 1986. Imprimé.

---. (trad. et éd.). Les Sagas islandaises. Paris : Gallimard NRF, Pléiade, 1987 (1 ${ }^{\mathrm{e}}$ édition). Imprimé.

Chartier, Daniel. A Bibliography on the Imagined North: Arctic, Winter, Antarctic. Bibliographie sur l'imaginaire du Nord (Arctique, hiver, Antarctique). Bibliografi över föreställningen om det nordliga: Arktis, vinter, Antarktis. Ritaskrá um hið imyndaða norður : Norðurskautið, veturinn, Suðurskautið. Montréal: Presses de l'Université du Québec/Imaginaire | Nord, 2007. Imprimé.

Chartier, Daniel et Sumarliði R. Ísleifsson (dir.). Iceland and Images of the North. Montréal et Reykjavík: Presses de l'Université du Québec/ Imaginaire | Nord, 2011. Imprimé.

Chateaubriand, François-René. Les Martyrs, in Euvres romanesques et voyages, t. II. Paris : Gallimard NRF, Pléiade, 1969. Imprimé.

Dasent, George Webbe (trad.). The Story of Burnt Njal. Icelandic Saga Database, 1861. Web. 14 août 2016.

Diderot, Denis. Supplément au Voyage de Bougainville [...], édition numérique réalisée à partir du texte de 1772. Les Classiques des sciences sociales, 2002. Web. 13 août 2016.

Emion, François et Hanna Steinunn Thorleifsdottir (dir.). L'Islande dans l'imaginaire. Caen : Presses Universitaires de Caen, 2013. Imprimé.

Gautier, Théophile. Un Tour en Belgique et en Hollande, in Caprices et Zigzags. Paris : Victor Lecou, 1852 ( $1^{\mathrm{e}}$ édition). Imprimé. Consultable en ligne : http://gallica.bnf.fr/ark:/12148/bpt6k102024x.r=.langFR.

« Indigène. » Le Trésor de la Langue Française informatisée. Web. 11 août 2016. Labonne, Henry. L'Islande et l'archipel des Faroer. Paris : Librairie Hachette et Cie, $1888 \quad\left(1^{\mathrm{e}} \quad\right.$ édition). Imprimé. Consultable en ligne : https://books.google.ca/books?id=5P0NAAAAYAAJ.

«LECLERCQ Jules-Joseph. » Académie Royale des Sciences d'Outre-Mer. Biographie coloniale Belge, 1948. Web. 14 août 2016.

Leclercq, Jules (trad.). Mythologie scandinave. Légendes des sagas. rééd. PontAuthou: Ed. d'Héligoland, 2010 ( $1^{\mathrm{e}}$ édition : Paris : Ernest Lerou, 1886). Imprimé.

---. Aux chutes du Zambèze : Du Cap au Katanga. Paris : Librairie Pierre Roger, $1926 \quad\left(1^{\mathrm{e}} \quad\right.$ édition). Imprimé. Consultable en ligne : http://gallica.bnf.fr/ark:/12148/bpt6k103878x.

---. Au pays de Paul et Virginie. Paris : E. Plon et Cie, 1895 ( $1^{\mathrm{e}}$ édition). Imprimé. Consultable en ligne : http://gallica.bnf.fr/ark:/12148/bpt6k2069453.

---. Du Caucase aux monts Alaï: Transcaspie, Boukharie, Ferganah. Paris : E. Plon et Cie, 1890 ( $1^{\mathrm{e}}$ édition). Imprimé. Consultable en ligne: https://books.google.ca/books?id=QkcEAAAAYAAJ. 
---. L'Islande et sa littérature. Bruxelles : Maurice Lamertin, 1923 ( $1^{\mathrm{e}}$ édition). Imprimé. Consultable en ligne : https://books.google.com/books/.../L_Islande_et_sa_littérature.html.

---. La Terre de Glace. Féroë, Islande, les geysers, le Mont Hekla. Paris : E. Plon et Cie, 1883 ( $1^{\mathrm{e}}$ édition). Imprimé. Consultable en ligne : https://archive.org/details/8SCSUP87606NOR.

---. La Terre des merveilles : promenade au parc national de l'Amérique du Nord. Paris : Librairie Hachette et Cie, 1886 ( $1^{\mathrm{e}}$ édition). Imprimé. Consultable en ligne : http://gallica.bnf.fr/ark:/12148/bpt6k408967h.

---. La Vallée des geysers. Paris : Plon, 1899. Imprimé. Consultable en ligne : http://gallica.bnf.fr/ark:/12148/bpt6k116572s.

---. Un été en Amérique, de l'Atlantique aux Montagnes Rocheuses. Paris : E. Plon et Cie, 1877 ( $1^{\mathrm{e}}$ édition). Imprimé. Consultable en ligne : https://books.google.ca/books?id=aLETAAAAYAAJ.

---. Voyage aux îles Fortunées, le pic de Ténériffe et les Canaries. Paris : E. Plon et Cie, $1880 \quad\left(1^{\mathrm{e}}\right.$ édition). Imprimé. Consultable en ligne : http://gallica.bnf.fr/ark:/12148/bpt6k1483568.r=.

---. Voyages dans le Nord de l'Europe : Norwège et Laponie (1871-1873). Tours : Alfred Mame et fils, 1875 ( $1^{\mathrm{e}}$ édition). Imprimé. Consultable en ligne : http://gallica.bnf.fr/ark:/12148/bpt6k431959q.

Loti, Pierre. Suprêmes Visions d'Orient (Constantinople et la Thrace 1910-1913), in Voyages (1872-1913). Paris : Robert Laffont, 1991 (1édition : 1921). Imprimé.

Marmier, Xavier. Lettres sur l'Islande. Paris : Félix Bonnaire, 1837 (1 $1^{\mathrm{e}}$ édition). Imprimé. Consultable en ligne : https://books.google.ca/books/about/Lettres_sur_l_Islande.html?id=32VAA AAAYAAJ\&redir_esc $=\mathrm{y}$.

« Merveille. » Le Trésor de la Langue Française informatisée. Web. 11 août 2016. Montesquieu, Baron de. De l'Esprit des lois I. Paris : GF Flammarion, $1979\left(1^{\mathrm{e}}\right.$ édition : 1748). Imprimé.

Oslund, Karen. Iceland imagined. Nature, culture, and storytelling in the North Atlantic. Seattle et Londres : University of Washington Press, 2011. Imprimé.

Polet, Jean-Claude (dir.). "Leclerc (Jules). 1848-1928". Patrimoine littéraire européen : Anthologie en langue française. Index général. Bruxelles : De Boeck Université, 2000, p. 452.

Rousseau, Jean-Jacques. Discours sur l'origine et les fondements de l'inégalité parmi les hommes. Paris : Gallimard Folio, 1969 ( $1^{\mathrm{e}}$ édition : 1755). Imprimé.

Segalen, Victor. Essai sur l'exotisme. Montpellier : Fata Morgana, 1978 ( $1^{\mathrm{e}}$ édition, posthume). Imprimé.

Staël-Holstein, Mme de. De la littérature. Paris : GF Flammarion, 1991 (1édition : 1800). Imprimé.

Wawn, Andrew. The Vikings and the Victorians. Inventing the Old North in Nineteenth-Century Britain. Cambridge : D. S. Brewer, 2000. Imprimé. 